\title{
On the Quality of Wireless Network Connectivity
}

\author{
Soura Dasgupta \\ Department of Electrical and Computer Engineering \\ The University of Iowa
}

\author{
Guoqiang Mao \\ School of Electrical and Information Engineering \\ The University of Sydney \\ National ICT Australia
}

\begin{abstract}
Despite intensive research in the area of network connectivity, there is an important category of problems that remain unsolved: how to measure the quality of connectivity of a wireless multi-hop network which has a realistic number of nodes, not necessarily large enough to warrant the use of asymptotic analysis, and has unreliable connections, reflecting the inherent unreliable characteristics of wireless communications? The quality of connectivity measures how easily and reliably a packet sent by a node can reach another node. It complements the use of capacity to measure the quality of a network in saturated traffic scenarios and provides a native measure of the quality of (end-to-end) network connections. In this paper, we explore the use of probabilistic connectivity matrix as a possible tool to measure the quality of network connectivity. Some interesting properties of the probabilistic connectivity matrix and their connections to the quality of connectivity are demonstrated. We argue that the largest eigenvalue of the probabilistic connectivity matrix can serve as a good measure of the quality of network connectivity.
\end{abstract}

Index Terms-Connectivity, network quality, probabilistic connectivity matrix

\section{INTRODUCTION}

Connectivity is one of the most fundamental properties of wireless multi-hop networks [1], [2], [3], and is a prerequisite for providing many network functions, e.g. routing, scheduling and localization. A network is said to be connected if and only if (iff) there is a (multi-hop) path between any pair of nodes. Further, a network is said to be $k$-connected iff there are $k$ mutually independent paths between any pair of nodes that do not share any node in common except the starting and the ending nodes. $k$ connectivity is often required for robust operations of the network.

This research is partially supported by US NSF grants ECS-0622017, CCF-072902, and CCF-0830747.

This research is partially supported by ARC Discovery project DP110100538 and by the Air Force Research Laboratory, under agreement number FA2386-10-1-4102. The U.S. Government is authorized to reproduce and distribute reprints for Governmental purposes notwithstanding any copyright notation thereon. The views and conclusions contained herein are those of the authors and should not be interpreted as necessarily representing the official policies or endorsements, either expressed or implied, of the Air Force Research Laboratory or the U.S Government.
There are two general approaches to studying the connectivity problem. The first, spearheaded by the seminal work of Penrose [3] and Gupta and Kumar [1], is based on an asymptotic analysis of large-scale random networks, which considers a network of $n$ nodes that are i.i.d. on an area with an underlying uniform distribution. A pair of nodes are directly connected iff their Euclidean distance is smaller than or equal to a given threshold $r(n)$, independent of other connections. Some interesting results are obtained on the value of $r(n)$ required for the above network to be asymptotically almost surely connected as $n \rightarrow \infty$. In [4], [5], the authors extended the above results by Penrose and Gupta and Kumar from the unit disk model to a random connection model, in which any pair of nodes separated by a displacement $\boldsymbol{x}$ are directly connected with probability $g(\boldsymbol{x})$, independent of other connections. The analytical techniques used in this approach have some intrinsic connections to continuum percolation theory [6] which is usually based on a network setting with nodes Poissonly distributed in an infinite area and studies the conditions required for the network to have a connected component containing an infinite number of nodes (in other words, the network percolates). We refer readers to [7] for a more comprehensive review of work in the area.

The second approach is based on a deterministic setting and studies the connectivity and other topological properties of a network using algebraic graph theory. Specifically, consider a network with a set of $n$ nodes. Its property can be studied using its underlying graph $G(V, E)$, where $V \triangleq\left\{v_{1}, \ldots, v_{n}\right\}$ denotes the vertex set and $E$ denotes the edge set. The underlying graph is obtained by representing each node in the network uniquely using a vertex and the converse. An undirected edge exists between two vertices iff there is a direct connection (or link) between the associated nodes 1 . Define an adjacency matrix $A_{G}$ of the graph $G(V, E)$ to be a symmetric $n \times n$ matrix whose $(i, j)^{t h}, i \neq j$ entry is equal to one if there is an edge between $v_{i}$ and $v_{j}$ and is equal to zero otherwise. Further,

\footnotetext{
${ }^{1}$ In this paper, we limit our discussions to a simple graph (network) where there is at most one edge (link) between a pair of vertices (nodes) and an undirected graph.
} 
the diagonal entries of $A_{G}$ are all equal to zero. The eigenvalues of the graph $G(V, E)$ are defined to be the eigenvalues of $A_{G}$. The network connectivity information, e.g. connectivity and $k$-connectivity, is entirely contained in its adjacency matrix. Many interesting connectivity and topological properties of the network can be obtained by investigating the eigenvalues of its underlying graph. For example, let $\mu_{1} \geq \ldots \geq \mu_{n}$ be the eigenvalues of a graph $G$. If $\mu_{1}=\mu_{2}$, then $G$ is disconnected. If $\mu_{1}=-\mu_{n}$ and $G$ is not empty, then at least one connected component of $G$ is nonempty and bipartite [8, p. 28-6]. If the number of distinct eigenvalues of $G$ is $r$, then $G$ has a diameter of at most $r-1$ [9]. Some researchers have also studied the properties of the underlying graph using its Laplacian matrix [10], where the Laplacian matrix of a graph $G$ is defined as $L_{G} \triangleq D-A_{G}$ and $D$ is a diagonal matrix with degrees of vertices in $G$ on the diagonal. Particularly, the algebraic connectivity of a graph $G$ is the secondsmallest eigenvalue of $L_{G}$ and it is greater than 0 iff $G$ is a connected graph. We refer readers to [9] and [11] for a comprehensive treatment of the topic. Reference [8] provides a concise summary of major results in the area.

The most related research to the work to be presented in this paper is possibly the more recent work of Brooks et al. [12]. In [12] Brooks et al. considered a probabilistic version of the adjacency matrix and defined a probabilistic adjacency matrix as a $n \times n$ square matrix $M$ whose $(i, j)^{t h}$ entry $m_{i j}$ represents the probability of having a direct connection between distinct nodes $i$ and $j$, and $m_{i i}=0$. They established that the probability that there exists at least one walk of length $z$ between nodes $i$ and $j$ is $m_{i j}^{z}$, where $m_{i j}^{z}$ is the $(i, j)^{t h}$ entry of $M \otimes M \otimes \cdots \otimes M(z$ times $)$. Here $C \triangleq A \otimes B$ is defined by $C_{i j}=1-\prod_{l \neq i, j}\left(1-A_{i l} B_{l j}\right)$ where $A_{i j}, B_{i j}$ and $C_{i j}$ are the $(i, j)^{t h}$ entries of the $n \times n$ square matrix $A, B$ and $C$ respectively. A walk of length $z$ between nodes $i$ and $j$ is a sequence of $z$ edges, where the first edge starts at $i$, the last edge ends at $j$, and the starting vertex of each intermediate edge is the ending vertex of its preceding edge. A path of length $z$ between nodes $i$ and $j$ is a walk of length $z$ in which the edges are distinct.

Despite intensive research in the area, there is an important category of problems that remain unsolved: how to measure the quality of connectivity of a wireless multihop network which has a realistic number of nodes, not necessarily large enough to warrant the use of asymptotic analysis, and has unreliable connections, reflecting the inherent unreliable characteristics of wireless communications? The quality of connectivity measures how easily and reliably a packet sent by a node can reach another node. It complements the use of capacity to measure the quality of a network in saturated traffic scenarios and provides a native measure of the quality of (end-to-end) network connections. In the following paragraphs, we elaborate on the above question using using two examples.

Example 1: Consider a network with a fixed number of nodes with known transmission power to be deployed in a certain environment. Assume that the wireless propagation model in that environment is known and its characteristics have been quantified through a priori measurements or empirical estimation. Further, a link exists between two nodes iff the received signal strength from one node at the other node, whose propagation follows the wireless propagation model, is greater than or equal to a predetermined threshold and the same is also true in the opposite direction. One can then find the probability that a link exists between two nodes at two fixed locations: It is determined by the probability that the received signal strength is greater than or equal to the pre-determined threshold. Two related questions can be asked: a) If these nodes are deployed at a set of known locations, what is the quality of connectivity of the network, measured by the probability that there is a path between any two nodes, as compared to node deployment at another set of locations? b) How to optimize the node deployment to maximize the quality of connectivity?

Example 2: Consider a network with a fixed number of nodes. The transmission between a pair of nodes with a direct connection, say $v_{i}$ and $v_{j}$, may fail with a known probability, say $a_{i j}$, quantifying the inherent unreliable characteristics of wireless communications. There are no direct connections between some pairs of nodes because the probability of successful transmission between them is too low to be acceptable. How to measure the quality of connectivity of such a network, in the sense that a packet transmitted from one node can easily and reliably reach another node via a multi-hop path. Will a single "good" path between a pair of nodes be more preferable than multiple "bad" paths? These are further illustrated using Fig. 1 and 2 .

In this paper, we explore the use of probabilistic connectivity matrix, a concept to be defined later in Section II as a possible tool to measure the quality of network connectivity. Some interesting properties of the probabilistic connectivity matrix and their connections to the quality of connectivity are demonstrated.

The rest of the paper is organized as follows. Section III defines the network settings, the probabilistic connectivity matrix and gives a method to compute the matrix. Section III introduces certain inequalities associated with the entries of the probabilistic connectivity matrix. Section IV] proves several important results about the probabilistic connectivity matrix. These directly associate the largest eigenvalue of the probabilistic connectivity matrix to the quality of connectivity and expose a structure that holds the promise of facilitating associated optimization tasks. Section $[\mathrm{V}$ concludes the paper and discusses future work. 


$\begin{array}{llllllll}0.9 & & 0.8 & & 0.95 & 0.85 & \circ \\ v_{1} & v_{2} & & v_{3} & & v_{4} & v_{5}\end{array}$

(a)

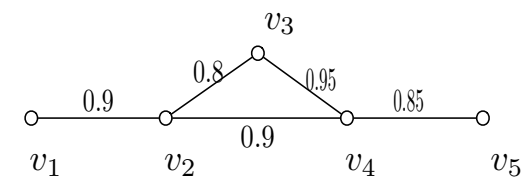

(b)

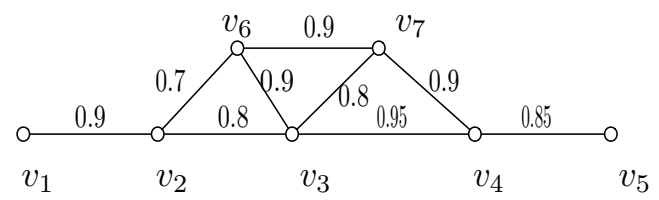

(c)

Figure 1. An illustration of networks with different quality of connectivity. A solid line represents a direct connection between two nodes and the number beside the line represents the corresponding transmission successful probability. The networks shown in (a), (b), and (c) are all connected networks but not 2-connected networks, i.e. their connectivity cannot be differentiated using the k-connectivity concept. However intuitively the quality of the network in (b) is better than that of the network in (a) because of the availability of the additional highquality link between $v_{2}$ and $v_{4}$ in (b). The quality of the network in (c) is even better because of the availability of the additional nodes and the associated high-quality links, hence additional routes, if these additional nodes act as relay nodes only. If these additional nodes also generate their own traffic, it is uncertain whether the quality of the network in (c) is better or not. Therefore it is important to develop a measure to quantitatively compare the quality of connectivity (for the networks in (a) and (b)) and to evaluate the benefit of additional nodes on connectivity (for the network in (c)).

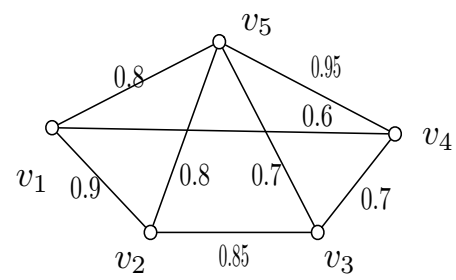

(a)

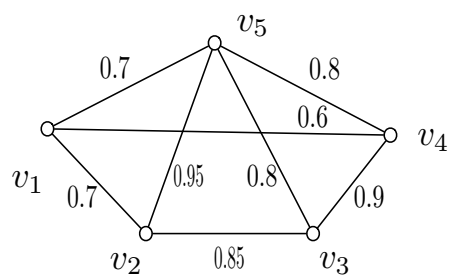

(b)

Figure 2. The networks shown in (a) and (b) have the same topology but different link quality. It is difficult to compare the quality of the two networks.

\section{Definition And CONSTRUCTion OF The PRoBABILISTIC CONNECTIVITY MATRIX}

In this section we define the network to be studied, its probabilistic adjacency matrix and probabilistic connectivity matrix, and gives an approach to computing the probabilistic connectivity matrix.

Consider a network of $n$ nodes. For some pair of nodes, an edge (or link) may exist with a non-negligible probability. The edges are considered to be undirected. That is, if a node $v_{i}$ is connected to a node $v_{j}$, then the node $v_{j}$ is also connected to the node $v_{i}$. Further, it is assumed that the event that there is an edge between a pair of nodes and the event that there is an edge between another distinct pair of nodes are independent.

Denote the underlying graph of the above network by $G(V, E)$, where $V=\left\{v_{1}, \ldots, v_{n}\right\}$ is the vertex set and $E=\left\{e_{1}, \ldots, e_{m}\right\}$ is the edge set, which contains the set of all possible edges. Here both the vertices and the edges are indexed from 1 to $n$ and from 1 to $m$ respectively. For convenience, in some parts of this paper we also use the symbol $e_{i j}$ to denote an edge between vertices $v_{i}$ and $v_{j}$ when there is no confusion. We associate with each edge $e_{i}, i \in\{1, \ldots m\}$, an indicator random variable $I_{i}$ such that $I_{i}=1$ if the edge $e_{i}$ exists; $I_{i}=0$ if the edge $e_{i}$ does not exist. The indicator random variables $I_{i j}, i \neq j$ and $i, j \in\{1, \ldots n\}$, are defined analogously.

In the following, we give a definition of the probabilistic adjacency matrix:

Definition 1: The probabilistic adjacency matrix of $G(V, E)$, denoted by $A_{G}$, is a $n \times n$ matrix such that its $(i, j)^{t h}, i \neq j$, entry $a_{i j} \triangleq \operatorname{Pr}\left(I_{i j}=1\right)$ and its diagonal entries are all equal to 1 .

Due to the undirected property of an edge mentioned above, $A_{G}$ is a symmetric matrix, i.e. $a_{i j}=a_{j i}$. Note that the diagonal entries of $A_{G}$ are defined to be 1 , which is different from that common in the literature. In [13] we have discussed the implication of this definition in the context of mobile ad-hoc networks. This treatment of the diagonal entries can be associated with the fact that a node in the network can keep a packet until better transmission opportunity arises when it finds the wireless channel busy.

The probabilistic connectivity matrix is defined in the following:

Definition 2: The probabilistic connectivity matrix of $G(V, E)$, denoted by $Q_{G}$, is a $n \times n$ matrix such that its $(i, j)^{t h}, i \neq j$, entry is the probability that there exists a path between vertices $v_{i}$ and $v_{j}$, and its diagonal entries are all equal to 1 .

As a ready consequence of the symmetry of $A_{G}, Q_{G}$ is also a symmetric matrix.

Given the probabilistic adjacency matrix $A_{G}$, the probabilistic connectivity matrix $Q_{G}$ is fully determined. However the computation of $Q_{G}$ is not trivial because for a pair of vertices $v_{i}$ and $v_{j}$, there may be multiple paths between 
them and some of them may share common edges, i.e. are not independent. In the following paragraph, we give an approach to computing the probabilistic connectivity matrix.

Let $\left(I_{1}, \ldots, I_{m}\right)$ be a particular instance of the indicator random variables associated with an instance of the random edge set. Let $Q_{G} \mid\left(I_{1}, \ldots, I_{m}\right)$ be the connectivity matrix of $G$ conditioned on $\left(I_{1}, \ldots, I_{m}\right)$. The $(i, j)^{t h}$ entry of $Q_{G} \mid\left(I_{1}, \ldots, I_{m}\right)$ is either 0 , when there is no path between $v_{i}$ and $v_{j}$, or 1 when there exists a path between $v_{i}$ and $v_{j}$ (see also Lemma 7 in Appendix I). The diagonal entries of $Q_{G} \mid\left(I_{1}, \ldots, I_{m}\right)$ are always 1 . Conditioned on $\left(I_{1}, \ldots, I_{m}\right), G(V, E)$ is just a deterministic graph. Therefore the entries of $Q_{G} \mid\left(I_{1}, \ldots, I_{m}\right)$ can be efficiently computed using a search algorithm, such as breadth-first search. Given $Q_{G} \mid\left(I_{1}, \ldots, I_{m}\right), Q_{G}$ can be computed using the following equation:

$$
Q_{G}=E\left(Q_{G} \mid\left(I_{1}, \ldots, I_{m}\right)\right)
$$

where the expectation is taken over all possible instances of $\left(I_{1}, \ldots, I_{m}\right)$.

The approach suggested in the last paragraph is essentially a brute-force approach to computing $Q_{G}$. We expect that more efficient algorithms can be designed to compute $Q_{G}$. However the main focus of the paper is on exploring the properties of $Q_{G}$ that facilitate the connectivity analysis and an extensive discussion of the algorithms to compute $Q_{G}$ is beyond the scope of the paper.

Remark 1: For simplicity, the terms used in our discussion are based on the problems in Example 11. The discussion however can be easily adapted to the analysis of the problems in Example 2. For example, if $a_{i j}$ is defined to be the probability that a transmission between nodes $v_{i}$ and $v_{j}$ is successful, the $(i, j)^{t h}$ entry of the probabilistic connectivity matrix $Q_{G}$ computed using (1) then gives the probability that a transmission from $v_{i}$ to $v_{j}$ via a multi-hop path is successful under the best routing algorithm, which can always find a shortest and error-free path between from $v_{i}$ to $v_{j}$ if it exists, or alternatively, the probability that a packet broadcast from $v_{i}$ can reach $v_{j}$ where each node receiving the packet only broadcasts the packet once. Therefore the $(i, j)^{t h}$ entry of $Q_{G}$ can be used as a quality measure of the end-to-end paths between $v_{i}$ and $v_{j}$, which takes into account the fact that availability of an extra path between a pair of nodes can be exploited to improve the probability of successful transmissions.

\section{Some Key Inequalities for Connection PROBABILITIES}

The entries of the probabilistic connectivity matrix give an intuitive idea about the overall quality of end-to-end paths in a network. In this section, we provide some important inequalities that may facilitate the analysis of the quality of connectivity. Some of these inequalities are exploited in the next section to establish some key properties of the probabilistic connection matrix itself.

We first introduce some concepts and results that are required for the further analysis of the probabilistic connectivity matrix $Q_{G}$.

For a random graph with a given set of vertices, a particular event is increasing if the event is preserved when more edges are added into the graph. An event is decreasing if its complement is increasing.

The following theorems on FKG inequality and BK inequality respectively are used:

Theorem 1: [6, Theorem 1.4] (FKG Inequality) If events $A$ and $B$ are both increasing events or decreasing events depending on the state of finitely many edges, then

$$
\operatorname{Pr}(A \cap B) \geq \operatorname{Pr}(A) \operatorname{Pr}(B)
$$

Theorem 2: [14], [6, Theorem 1.5] (BK Inequality) If events $A$ and $B$ are both increasing events depending on the state of finitely many edges, then

$$
\operatorname{Pr}(A \square B) \leq \operatorname{Pr}(A) \operatorname{Pr}(B)
$$

where for two events $A$ and $B, A \square B$ denotes the event that there exist two disjoint sets of edges such that the first set of edges guarantees the occurrence of $A$ and the second set of edges guarantees the occurrence of $B$.

There is a recent extension of Theorem 2 to two arbitrary events, i.e. events $A$ and $B$ in Theorem 2 do not have to be increasing events [15].

Denote by $\xi_{i j}$ the event that there is a path between vertices $v_{i}$ and $v_{j}, i \neq j$. Denote by $\xi_{i k j}$ the event that there is a path between vertices $v_{i}$ and $v_{j}$ and that path passes through the third vertex $v_{k}$, where $k \in \Gamma_{n} \backslash\{i, j\}$ and $\Gamma_{n}$ is the set of indices of all vertices. Denote by $\eta_{i j}$ the event that there is an edge between vertices $v_{i}$ and $v_{j}$. Denote by $\pi_{i k j}$ the event that there is a path between vertices $v_{i}$ and $v_{k}$ and there is a path between vertices $v_{k}$ and $v_{j}$, where $k \in \Gamma_{n} \backslash\{i, j\}$. Obviously

$$
\pi_{i k j} \Rightarrow \xi_{i j}
$$

It can also be shown from the above definitions that

$$
\xi_{i j}=\eta_{i j} \cup\left(\cup_{k \neq i, j} \xi_{i k j}\right)
$$

Let $q_{i j}, i \neq j$, be the $(i, j)^{t h}$ entry of $Q_{G}$, i.e. $q_{i j}=$ $\operatorname{Pr}\left(\xi_{i j}\right)$. The following lemma can be readily obtained from the FKG inequality and the above definitions

Lemma 1: For two distinct indices $i, j \in \Gamma_{n}$ and $\forall k \in$ $\Gamma_{n} \backslash\{i, j\}$

$$
q_{i j} \geq \max _{k \in \Gamma_{n} \backslash\{i, j\}} q_{i k} q_{k j}
$$

Proof: It follows readily from the above definitions that the event $\xi_{i j}$ is an increasing event. Due to (2) and the FKG inequality:

$\operatorname{Pr}\left(\xi_{i j}\right) \geq \operatorname{Pr}\left(\pi_{i k j}\right)=\operatorname{Pr}\left(\xi_{i k} \cap \xi_{k j}\right) \geq \operatorname{Pr}\left(\xi_{i k}\right) \operatorname{Pr}\left(\xi_{k j}\right)$ 
The conclusion follows.

Lemma 1 gives a lower bound of $q_{i j}$. The following lemma gives an upper bound of $q_{i j}$ :

Lemma 2: For two distinct indices $i, j \in \Gamma_{n}$ and $\forall k \in$ $\Gamma_{n} \backslash\{i, j\}$,

$$
q_{i j} \leq 1-\left(1-a_{i j}\right) \prod_{k \in \Gamma_{n} \backslash\{i, j\}}\left(1-q_{i k} q_{k j}\right)
$$

where $a_{i j}=\operatorname{Pr}\left(\eta_{i j}\right)$.

Proof: We will first show that $\xi_{i k j} \Leftrightarrow \xi_{i k} \square \xi_{k j}$. That is, the occurrence of the event $\xi_{i k j}$ is a sufficient and necessary condition for the occurrence of the event $\xi_{i k} \square \xi_{k j}$.

Using the definition of $\xi_{i k j}$, occurrence of $\xi_{i k j}$ means that there is a path between vertices $v_{i}$ and $v_{j}$ and that path passes through vertex $v_{k}$. It follows that there exist a path between vertex $i$ and vertex $v_{k}$ and a path between vertex $v_{k}$ and vertex $v_{j}$ and the two paths do not have edge(s) in common. Otherwise, it will contradict the definition of $\xi_{i k j}$, particularly as the definition of a path requires the edges to be distinct. Therefore $\xi_{i k j} \Rightarrow \xi_{i k} \square \xi_{k j}$. Likewise, $\xi_{i k j} \Leftarrow \xi_{i k} \square \xi_{k j}$ also follows directly from the definitions of $\xi_{i k j}, \xi_{i k}, \xi_{k j}$ and $\xi_{i k} \square \xi_{k j}$. Consequently

$$
\operatorname{Pr}\left(\xi_{i k j}\right)=\operatorname{Pr}\left(\xi_{i k} \square \xi_{k j}\right) \leq \operatorname{Pr}\left(\xi_{i k}\right) \operatorname{Pr}\left(\xi_{k j}\right)
$$

where the inequality is a direct result of the BK inequality.

With a little bit abuse of the terminology, in the following derivations we also use $\xi_{i k j}$ to represent the set of edges that make the event $\xi_{i k j}$ happen, and use $\eta_{i j}$ to denote the edge between vertices $v_{i}$ and $v_{j}$.

Note that the set of edges $\cup_{k \in \Gamma_{n} \backslash\{i, j\}} \xi_{i k j}$ does not contain $\eta_{i j}$. Therefore using (3) and independence of edges (used in the third step)

$$
\begin{aligned}
q_{i j} & =\operatorname{Pr}\left(\eta_{i j} \cup\left(\cup_{k \in \Gamma_{n} \backslash\{i, j\}} \xi_{i k j}\right)\right) \\
& =1-\operatorname{Pr}\left(\overline{\eta_{i j}} \cap\left(\overline{\cup_{k \in \Gamma_{n} \backslash\{i, j\}} \xi_{i k j}}\right)\right) \\
& =1-\left(1-a_{i j}\right) \operatorname{Pr}\left(\cap_{k \in \Gamma_{n} \backslash\{i, j\}} \overline{\xi_{i k j}}\right) \\
& \leq 1-\left(1-a_{i j}\right) \prod_{k \in \Gamma_{n} \backslash\{i, j\}} \operatorname{Pr}\left(\overline{\xi_{i k j}}\right) \\
& =1-\left(1-a_{i j}\right) \prod_{k \in \Gamma_{n} \backslash\{i, j\}}\left(1-\operatorname{Pr}\left(\xi_{i k j}\right)\right) \\
& \leq 1-\left(1-a_{i j}\right) \prod_{k \in \Gamma_{n} \backslash\{i, j\}}\left(1-q_{i k} q_{k j}\right)
\end{aligned}
$$

where in (8), FKG inequality and the obvious fact that $\overline{\bar{\xi}_{i k j}}$ is a decreasing event are used and the last step results due to (7).

When there is no edge between vertices $v_{i}$ and $v_{j}$, which is the generic case, the upper and lower bounds in Lemmas 1 and 2 reduce to

$$
\max _{k \in \Gamma_{n} \backslash\{i, j\}} q_{i k} q_{k j} \leq q_{i j} \leq 1-\prod_{k \in \Gamma_{n} \backslash\{i, j\}}\left(1-q_{i k} q_{k j}\right)
$$

The above inequality sheds insight on how the quality of paths between a pair of vertices is related to the quality of paths between other pairs of vertices. It can be possibly used to determine the most effective way of improving the quality of a particular set of paths by improving the quality of a particular (set of) edge(s), or equivalently what can be reasonably expected from an improvement of a particular edge on the quality of end-to-end paths.

The following lemma further shows that relation among entries of the path matrix $Q_{G}$ can be further used to derive some topological information of the graph.

Lemma 3: If $q_{i j}=q_{i k} q_{k j}$ for three distinct vertices $v_{i}, v_{j}$ and $v_{k}$, the vertex set $V$ of the underlying graph $G(V, E)$ can be divided into three non-empty and nonintersecting sub-sets $V_{1}, V_{2}$ and $V_{3}$ such that $v_{i} \in V_{1}$, $v_{j} \in V_{3}$ and $V_{2}=\left\{v_{k}\right\}$ and any possible path between a vertex in $V_{1}$ and a vertex in $V_{2}$ must pass through $v_{k}$. Further, for any pair of vertices $v_{l}$ and $v_{m}$, where $v_{l} \in V_{1}$ and $v_{m} \in V_{3}, q_{l m}=q_{l k} q_{k m}$.

Proof: Using (5) in the second step, it follows that

$$
\begin{aligned}
q_{i j} & =\operatorname{Pr}\left(\left(\xi_{i j} \backslash \pi_{i k j}\right) \cup \pi_{i k j}\right) \\
& =\operatorname{Pr}\left(\xi_{i j} \backslash \pi_{i k j}\right)+\operatorname{Pr}\left(\pi_{i k j}\right) \\
& \geq \operatorname{Pr}\left(\xi_{i j} \backslash \xi_{i k j}\right)+q_{i k} q_{k j}
\end{aligned}
$$

Therefore $q_{i j}=q_{i k} q_{k j}$ implies that $\operatorname{Pr}\left(\xi_{i j} \backslash \pi_{i k j}\right)=0$ or equivalently $\xi_{i j} \Leftrightarrow \pi_{i k j}$

Further, $\operatorname{Pr}\left(\xi_{i j} \backslash \pi_{i k j}\right)=0$ implies that a possible path (i.e. a path with a non-zero probability) connecting $v_{i}$ and $v_{k}$ and a possible path connecting $v_{k}$ and $v_{j}$ cannot have any edge in common. Otherwise a path from $v_{i}$ to $v_{j}$, bypassing $v_{k}$, exists with a non-zero probability which implies $\operatorname{Pr}\left(\xi_{i j} \backslash \xi_{i k j}\right)>0$. The conclusion follows readily that if $q_{i j}=q_{i k} q_{k j}$ for three distinct vertices $v_{i}, v_{j}$ and $v_{k}$, the vertex set $V$ of the underlying graph $G(V, E)$ can be divided into three non-empty and non-overlapping sub-sets $V_{1}, V_{2}$ and $V_{3}$ such that $v_{i} \in V_{1}, v_{j} \in V_{3}$ and $V_{2}=\left\{v_{k}\right\}$ and a path between a vertex in $V_{1}$ and a vertex in $V_{2}$, if exists, must pass through $v_{k}$.

Further, for any pair of vertices $v_{l}$ and $v_{m}$, where $v_{l} \in$ $V_{1}$ and $v_{m} \in V_{3}$, it is easily shown that $\operatorname{Pr}\left(\xi_{l m} \backslash \pi_{l k m}\right)=$ 0 . Due to independence of edges and further using the fact that $\operatorname{Pr}\left(\xi_{l m} \backslash \pi_{l k m}\right)=0$, it can be shown that

$$
\begin{aligned}
\operatorname{Pr}\left(\xi_{l m}\right)=\operatorname{Pr}\left(\pi_{l k m}\right) & =\operatorname{Pr}\left(\xi_{l k} \cap \xi_{k m}\right) \\
& =\operatorname{Pr}\left(\xi_{l k}\right) \operatorname{Pr}\left(\xi_{k m}\right)
\end{aligned}
$$

where (11) results due to the fact that under the condition of $\operatorname{Pr}\left(\xi_{l m} \backslash \pi_{l k m}\right)=0$, a path between vertices $v_{l}$ and $v_{k}$ and a path between vertices $v_{k}$ and $v_{m}$ cannot possibly have any edge in common.

An implication of Lemma 3 is that for any three distinct vertices, $v_{i}, v_{j}$ and $v_{k}$, if a relationship $q_{i j}=q_{i k} q_{k j}$ holds, vertex $v_{k}$ must be a critical vertex whose removal will render the graph disconnected. 


\section{Properties of the Connectivity Matrix}

Having established some inequalities obeyed by the entries of the probabilistic connectivity matrix $Q_{G}$, we now turn to establishing a measure of the quality of network connectivity. At the core of the development in this section is the following result.

Lemma 4: Each off-diagonal entry of the probabilistic connectivity matrix $Q_{G}$ is a multiaffine ${ }^{2}$ function of $a_{i j}$, $i \in\{1, \ldots, n\}, j>i$.

Proof: Observe that $a_{i j}=\operatorname{Pr}\left(\eta_{i j}\right)$ and the events $\eta_{i j}$, $i \in\{1, \ldots, n\}, j>i$ are independent. The conclusion in the lemma follows readily from the fact that the event associated with each $q_{i j}$, i.e. there exists a path between vertices $v_{i}$ and $v_{j}$, is a union of intersections of these events $\eta_{i j}, i \in\{1, \ldots, n\}, j>i$.

Not only does the multiaffine structure facilitate the proof of the main result below, we comment later in Remark 2, on how it is potentially useful for performing some of the optimization tasks inherent in maximizing connectivity.

A very desirable property of $Q_{G}$ is established below.

Theorem 3: The probabilistic connectivity matrix $Q_{G}$, defined for the vertex set $V=\left\{v_{1}, \cdots, v_{n}\right\}$, is a positive semi-definite matrix. Further, $Q_{G}$ is positive semidefinite but not positive definite iff there exist distinct $i, j \in\{1, \cdots, n\}$, such that $q_{i j}=1$.

Proof: See Appendix I.

Let $\lambda_{1} \geq \ldots \geq \lambda_{n}$ be the eigenvalues of $Q_{G}$. Note that $\lambda_{1}+\cdots+\lambda_{n}=n$. As an easy consequence of Theorem 3. $n \geq \lambda_{1} \geq 1$ and $1 \geq \lambda_{n} \geq 0$. In the best case, $Q_{G}$ is a matrix with all entries equal to 1 . Then $\lambda_{1}=n$ and $\lambda_{2}=\cdots=\lambda_{n}=0$. In the worst case, $Q_{G}$ is an identity matrix. Then $\lambda_{1}=\cdots=\lambda_{n}=1$. This suggests that $\lambda_{1}$, i.e. the largest eigenvalue of $Q_{G}$, can be used as a measure of quality of network connectivity and a larger $\lambda_{1}$ indicates a better quality.

We will make this idea more concrete in the following analysis. We start our discussion with a connected network and then extend to more generic cases. We will call a network connected if for all $i, j \in\{1, \cdots, n\}$, $q_{i j}>0$. Obviously the probabilistic connectivity matrix of a connected network is irreducible [16, p. 374] as all the entries of the matrix are non-zero. As a measure of the quality of network connectivity, if the path probabilities $q_{i j}$ increase, the largest eigenvalue of the probabilistic connectivity matrix should also increase. This is formally stated in the following theorem:

Theorem 4: Let $G(V, E)$ and $G^{\prime}\left(V, E^{\prime}\right)$ be the underlying graphs of two connected networks defined on the same vertex set $V$ but with different link probabilities. Let $Q_{G}$ and $Q_{G^{\prime}}$ be the probabilistic connectivity matrices of $G$ and $G^{\prime}$ respectively and let $\lambda_{\max }\left(Q_{G}\right)$ and $\lambda_{\max }\left(Q_{G^{\prime}}\right)$

\footnotetext{
${ }^{2} \mathrm{~A}$ multiaffine function is affine in each variable when the other variables are fixed.
}

be the largest eigenvalues of $Q_{G}$ and $Q_{G^{\prime}}$ respectively. If $Q_{G}^{\prime}-Q_{G}$ is a non-zero, non-negative matrix ${ }^{3}$, then

$$
\lambda_{\max }\left(Q_{G}\right)<\lambda_{\max }\left(Q_{G^{\prime}}\right)
$$

Proof: See Appendix II

After having analyzed the situation for connected networks, we now move on to the discussion of disconnected networks and show that the largest eigenvalue of the probabilistic connectivity matrix of a component, a concept defined in the next paragraph, provides a good measure of the quality of connectivity of that component. We will analyze two basic situations. Results for more complicated scenarios can be readily obtained from these results and Theorem 4, which applies to a connected network.

If the network is not connected, i.e. some entries of its probabilistic connectivity matrix is 0 , it can be easily shown that the network can be decomposed into disjoint components. A component is a maximal set of vertices where the probability that there is a path between any pair of vertices in the component is greater than zero. Two components are said to be disjoint if the probabilities that there is a path between any vertex in the first component and any vertex in the second component are all zeros.

Let the total number of components in the network be $k$ and the number of nodes in the $i^{t h}, 1 \leq i \leq k$, component be $n_{i}$. Further, denote the vertex set of the $i^{t h}$ component by $V_{i}$ and denote the subgraph induced on $V_{i}$ by $G_{i}$. Without loss of generality, we assume that the nodes in the network are properly labeled such that

$$
V_{i}=\left\{v_{\sum_{j=1}^{i-1} n_{j}+1}, \cdots, v_{\sum_{j=1}^{i-1} n_{j}+n_{i}}\right\}
$$

Let $Q_{G_{i}}$ be the probabilistic connectivity matrix of $G_{i}$. It follows that the probabilistic connectivity matrix of the network $Q_{G}$ can be expressed in the form of $Q_{G_{i}}, 1 \leq$ $i \leq k$, as

$$
Q_{G}=\operatorname{diag}\left\{Q_{G_{1}}, \cdots, Q_{G_{k}}\right\}
$$

and

$$
\lambda_{\max }\left(Q_{G}\right)=\max \left\{\lambda_{\max }\left(Q_{G_{1}}\right), \cdots, \lambda_{\max }\left(Q_{G_{k}}\right)\right\}
$$

We consider two basic situations: a) there are increases in some entries of $Q_{G}$ from non-zero values but such increases do not change the number of components in the network; b) there are increases in some entries of $Q_{G}$ from zero to non-zero values and such increases reduce the number of components in the network.

Under situation a), the vertex set of each component does not change. Let $Q_{G_{i}}$ be the probabilistic connectivity matrix of a component whose path probabilities have been increased and let $Q_{G_{i}^{\prime}}$ be the probabilistic connectivity matrix of the component after the change in path probabilities. Obviously $Q_{G_{i}^{\prime}}-Q_{G_{i}}$ is a non-zero, non-negative

\footnotetext{
${ }^{3} \mathrm{~A}$ matrix is non-negative if all its entries are greater than or equal to
} 
symmetric matrix. It then follows easily from Theorem 4 that $\lambda_{\max }\left(Q_{G_{i}^{\prime}}\right)>\lambda_{\max }\left(Q_{G_{i}}\right)$. Depending on whether $\lambda_{\max }\left(Q_{G_{i}^{\prime}}\right)$ is greater than $\lambda_{\max }\left(Q_{G}\right)$ or not however, the largest eigenvalue of the probabilistic connectivity matrix of the network may or may not increase.

We now move on to evaluate situation b) and consider a simplified scenario where increases in the path probabilities merge two originally disjoint components, denoted by $G_{i}$ and $G_{j}$. The more complicated scenario where increases in the path probabilities join more than two originally disjoint components can be obtained recursively as an extension of the above simplified scenario. Let $G^{\prime}$ be the underlying graph of the network after increases in path probabilities and let $G_{i j}^{\prime}$ be the subgraph in $G^{\prime}$ induced on the vertex set $V_{i} \cup V_{j}$. Obviously $Q_{G_{i j}^{\prime}}$ is an irreducible matrix and the following result can be established.

Lemma 5: Under the above settings,

$$
\lambda_{\max }\left(Q_{G_{i j}^{\prime}}\right)>\lambda_{\max }\left(\operatorname{diag}\left\{Q_{G_{i}}, Q_{G_{j}}\right\}\right)
$$

The proof of Lemma 5 is omitted due to space limitations.

Thus indeed the largest eigenvalues of the probabilistic connection matrices associated with disjoint components measure the quality of the components connection.

Remark 2: The fact that the largest eigenvalue of the probabilistic connectivity matrix measures connectivity, suggests the following obvious optimization. Modify one or more $a_{i j}$ under suitable constraints to maximize the largest eigenvalue of the probabilistic connectivity matrix. Results in [17] and [18] suggest that the multiaffine dependence of the $q_{i j}$ on the $a_{i j}$ together with the fact that $Q_{G}$ is positive semi-definite promise to facilitate such optimization.

\section{CONCLUSIONS AND FURTHER WORK}

In this paper we have explored the use of the probabilistic connectivity matrix as a possible tool to measure the quality of network connectivity. Some interesting properties of the probabilistic connectivity matrix and their connections to the quality of network connectivity were demonstrated. Particularly, the off-diagonal entries of the probabilistic connectivity matrix provide a measure of the quality of end-to-end connections and we have also provided theoretical analysis supporting the use of the largest eigenvalue of the probabilistic connectivity matrix as a measure of the quality of overall network connectivity. The analysis focused on the comparison of networks with the same number of nodes. For networks with different number of nodes, the largest eigenvalue of the probabilistic connectivity matrix normalized by the number of nodes may be used as the quality metric.

Inequalities between the entries of the probabilistic connectivity matrix were established. These may provide insights into the correlations between quality of end-toend connections. Further, the probabilistic connectivity matrix was shown to be a positive semi-definite matrix and its off-diagonal entries are multiaffine functions of link probabilities. These two properties are expected to be very helpful in optimization and robust network design, e.g. determining the link whose quality improvement will result in the maximum gain in network quality, and determining quantitatively the relative criticality of a link to either a particular end-to-end connection or to the entire network.

The results in the paper rely on two main assumptions: the links are symmetric and independent. We expect that our analysis can be readily extended such that the first assumption on symmetric links can be removed - in fact the results in Section III do not need this assumption. While in the asymmetric case the probabilistic connectivity matrix is no longer guaranteed to be positive semidefinite, we conjecture that the largest eigenvalue retains its significance. Discarding the second assumption requires more work. However, we are encouraged by the following observation. If we introduce conditional edge probabilities into the mix, then $Q_{G}$ is still a multiaffine function of the $a_{i j}$ and the conditional probabilities. Thus we still expect all the results in Section IV to hold, though the proof may be non-trivial. In real applications link correlations may arise due to both physical layer correlations and correlations caused by traffic congestion.

Another implicit assumption in the paper is that traffic is uniformly distributed and traffic between every sourcedestination pair is equally important. If this is not the case, a weighted version of the probabilistic connectivity matrix can be contemplated in which the entries of the matrix are weighted by a measure of the importance of the associated source-destination pairs. It remains to be investigated on whether our results can be extended to a weighted probabilistic connectivity matrix.

\section{APPENDIX I: PROOF OF THEOREM 3}

Let $a_{U}^{(n)}$ be the vector of $a_{i j}, i \neq j$ (remember that $a_{i j}$ is the probability that there is an edge between $v_{i}$ and $v_{j}$ ):

$$
a_{U}^{(n)} \triangleq\left[a_{i j}\right]_{i=1, j>i}^{n}
$$

Also define the set

$$
\Pi_{U}^{n}=\left\{a_{U}^{(n)} \mid 0 \leq a_{i j} \leq 1, i \in\{1, \ldots, n\}, j>i\right\}
$$

The corners of the above set are given by:

$$
\Pi_{U_{c}}^{n}=\left\{a_{U}^{(n)} \mid a_{i j} \in\{0,1\}, i \in\{1, \ldots, n\}, j>i\right\}
$$

These corners will play an important role in the subsequent development.

We observe that the positive semi-definiteness of a matrix is known to be a convex property, as defined in [18]. That is, if $A$ and $B$ are positive semi-definite then so is $(1-\alpha) A+\alpha B, \forall \alpha \in[0,1]$. In fact one can say a bit more: 
Lemma 6: Consider $n \times n$ matrices $A>0$ and $B \geq 0$. Then: $(1-\alpha) A+\alpha B>0, \forall \alpha \in[0,1)$

Proof: It is well known that there exists a matrix $H$, such that $H\left(A+A^{T}\right) H=I$ and for some diagonal $\Lambda$, $H\left(B+B^{T}\right) H=\Lambda \geq 0$. Then the result follows by noting that $(1-\alpha) I+\alpha \Lambda>0, \forall \alpha \in[0,1)$

Next we provide a key result that exploits the multiaffine dependence of $Q_{G}$ on the $a_{i j}$.

Proposition 1: Suppose $Q_{G}$ is positive semi-definite for all $a_{U}^{(n)} \in \Pi_{U_{c}}^{n}$. Then it is positive semi-definite for all $a_{U}^{(n)} \in \Pi_{U}^{n}$.

Proof: The combined use of Lemma 4 and Corollary 2.1 of [18] proves the result.

We must next show that $Q_{G}$ is positive semi-definite for all $a_{U}^{(n)} \in \Pi_{U_{c}}^{n}$. The following lemma is used in the proof of the conclusion that $Q_{G}$ is positive semi-definite for all $a_{U}^{(n)} \in \Pi_{U_{c}}^{n}$ :

Lemma 7: Suppose $a_{U}^{(n)} \in \Pi_{U_{c}}^{n}$, then for all $i, j, q_{i j} \in$ $\{0,1\}$.

Proof: When $a_{U}^{(n)} \in \Pi_{U_{c}}^{n}$, either there is an edge between vertices $v_{i}$ and $v_{j}$ surely when $a_{i j}=1$; or there is no edge between vertices $v_{i}$ and $v_{j}$ surely when $a_{i j}=$ 0 . The graph $G(V, E)$ becomes a deterministic graph. It follows that either there is a path between vertices $v_{i}$ and $v_{j}$ surely or there is no path between vertices $v_{i}$ and $v_{j}$ surely, i.e. for all $i, j, q_{i j} \in\{0,1\}$.

It can be further shown that the following lemma holds:

Lemma 8: Suppose for some distinct $i, j, q_{i j}=1$. Then row $i$ and row $j$ of $Q_{G}$ are identical, as are columns $i$ and $j$.

Proof: Note that $Q_{G}$ is a symmetric matrix. Thus it suffices to show that the row property holds. One has

$$
q_{i j}=q_{j i}=q_{i i}=q_{j j}=1
$$

Thus the $i^{\text {th }}$ and $j^{\text {th }}$ entries of the $i^{\text {th }}$ and $j^{\text {th }}$ rows are identical. Now consider any $k$ distinct from $i$ and $j$. Using Lemma 1 and (17):

$$
q_{i k} \geq q_{i j} q_{j k}=q_{j k} \text { and } q_{j k} \geq q_{i j} q_{i k}=q_{i k}
$$

It follows that: $q_{j k}=q_{i k}$.

We need one last lemma to complete the proof.

Lemma 9: Define $u_{m}$ to be a $m$-vector of all ones. Suppose $a_{U}^{(n)} \in \Pi_{U_{c}}^{n}$. Then for some $k$, there exist positive integers $m_{1}, \cdots, m_{k}$ whose sum is $n$, and a permutation matrix $P$, such that:

$$
Q_{G}=P \operatorname{diag}\left\{u_{m_{1}} u_{m_{1}}^{T}, \ldots, u_{m_{k}} u_{m_{k}}^{T}\right\} P^{T}
$$

Proof: We prove the lemma by induction.

As an easy consequence of Lemma 7, the result clearly holds for $n=2$ because when $n=2, Q_{G}$ is either equal to an identity matrix or equal to a matrix of all ones.

Now suppose that the lemma holds for all $n \leq m$. For convenience, we use also $Q_{n}$ to denote $Q_{G}$ when $|V|=n$.
When $n=m+1$, consider $Q_{m+1}$, corresponding to any $a_{U}^{(n)} \in \Pi_{U_{c}}^{n}$. Because of Lemma 7, all entries of $Q_{m+1}$ are in $\{0,1\}$. If $q_{i j}=0, \forall i \neq j$, then the results hold with $m_{i}=1$ and $k=n$. Now suppose there exists some distinct $i$ and $j$ for which $q_{i j}=1$. By symmetrically permuting $Q_{m+1}$, i.e. by relabeling the nodes if necessary, one can without loss of generality choose $\{i, j\}=\{1,2\}$. Choose $m_{l}$ to equal the number of ones in the first row of this possibly permuted $Q_{m+1}$. Through a further symmetric permutation/relabeling if necessary, without loss of generality one has:

$$
q_{1 j}=q_{j 1}=1, \forall j \in\left\{1, \ldots, m_{1}\right\}
$$

From Lemma 7

$$
q_{1 j}=q_{j 1}=0, \forall j \in\left\{m_{1}+1, \ldots, n\right\}
$$

From Lemma 8 , there holds:

$$
q_{i j}= \begin{cases}1 & \forall i, j \in\left\{1, \ldots, m_{1}\right\} \\ 0 & \forall i \in\left\{1, \ldots, m_{1}\right\} \text { and } j \in\left\{m_{1}+1, \ldots, n\right\} \\ 0 & \forall j \in\left\{1, \ldots, m_{1}\right\} \text { and } i \in\left\{m_{1}+1, \ldots, n\right\}\end{cases}
$$

Thus after relabeling one can express:

$$
Q_{G}=\operatorname{diag}\left\{u_{m_{1}} u_{m_{1}}^{T}, Q_{m+1-m_{1}}\right\}
$$

Further, there is no path from the vertex set $\left\{v_{1}, \ldots, v_{m_{1}}\right\}$ to the vertex set $\left\{v_{m_{1}}, \ldots, v_{n}\right\}$ and vice versa.

Obviously the entries of $Q_{m+1-m_{1}}$ form legitimate path probabilities with the vertex set $\left\{v_{m_{1}}, \ldots, v_{n}\right\}$. Then the inductive hypothesis proves the result.

We are now ready to prove Theorem 3

Observe the matrix in $(18)$ is positive semi-definite. Thus from Lemma $9, Q_{G}$ is positive semi-definite for all $a_{U}^{(n)} \in \Pi_{U_{c}}^{n}$. It then follows from Proposition 1 that $Q_{G}$ is positive semi-definite for all $a_{U}^{(n)} \in \Pi_{U}^{n}$. Therefore the first part of Theorem 3 that $Q_{G}$ is a positive semi-definite matrix is proved.

Now we proceed to prove the second part of Theorem 3 that $Q_{G}$ is positive semi-definite but not positive definite iff there exist distinct $i, j \in\{1, \cdots, n\}$, such that $q_{i j}=1$.

Suppose there exists distinct $i$ and $j$ such that $q_{i j}=1$. Then from Lemma 8 , at least two rows of $Q_{G}$ are identical. Thus $Q_{G}$ is singular and cannot be positive definite.

It remains to show that if for some $a_{U}^{(n)} \in \Pi_{U}^{n}$, all $q_{i j} \neq 1$ where $i \neq j$, then $Q_{G}$ is positive definite. We prove this by induction.

The result is clearly true for $n=2$. Suppose it holds for some $n=m$. Consider $n=m+1$.

To establish a contradiction suppose there is a $a_{U}^{(n)} \in$ $\Pi_{U}^{n}$ for which all $q_{i j} \neq 1$ where $i \neq j$, and yet $Q_{n}$ is not positive definite. This means all $a_{i j} \neq 1$ where $i \neq j$.

If for all $i \in\{1, \ldots, n-1\}, a_{i n}=0$, then for all $i \in\{1, \ldots, n-1\}, q_{\text {in }}=0$. Then vertex $v_{n}$ is disconnected from the vertex set $\left\{v_{1}, \ldots, v_{n-1}\right\}$ and for all $\{i, j\} \subseteq\{1, \ldots, n-1\}, q_{i j}$ are valid path probabilities 
in the subgraph induced on the vertex set $\left\{v_{1}, \ldots, v_{n-1}\right\}$. Further $Q_{n}=\operatorname{diag}\left\{Q_{n-1}, 1\right\}$. By hypothesis $Q_{n-1}$ is positive definite and thus so is $Q_{n}$. Because of the resulting contradiction with the hypothesis that $Q_{n}$ is not positive definite, it follows that for at least one $i \in\{1, \ldots, n-1\}$, $a_{\text {in }} \neq 0$.

Through relabeling, without sacrificing generality, assume that $a_{i n} \neq 0, \forall i \in\{1, \ldots, l\}$ and $a_{i n}=0, \forall i \in$ $\{l+1, \ldots, n-1\}$.

Define $R$ to be the probabilistic connectivity matrix obtained by keeping all corresponding $a_{i j}$ the same except $a_{l n}$, which is set to zero. Similarly, define $S$ to be the probabilistic connectivity matrix obtained by keeping all corresponding $a_{i j}$ the same except $a_{l n}$, which is set to 1 .

Observe, because of Lemma 4 that $Q_{n}$ is a convex combination of $R$ and $S$. Further, due to the hypothesis that $Q_{n}$ is not positive definite, both $R$ and $S$ are positive semi-definite but neither can be positive definite. Otherwise, using Lemma 6, $Q_{n}$ will be positive definite which leads to a contradiction of the hypothesis. In particular $R$ is positive semi-definite, but not positive definite. Now working with $R$, if $l>1$, then the probabilistic connectivity matrix obtained by keeping all corresponding $a_{i j}$ the same except $a_{2 n}$, which is set to zero, is similarly positive semi-definite, but not positive definite.

Working recursively in this fashion, the probabilistic connectivity matrix obtained by keeping all $a_{i j}$ the same except $a_{i n}=0, \forall i \in\{1, \ldots, n-1\}$, is positive semidefinite, but not positive definite. Thereby a contradiction is established with the conclusion obtained in the earlier paragraph that for at least one $i \in\{1, \ldots, n-1\}, a_{i n} \neq 0$.

\section{ApPendix II: Proof of Theorem 4}

The proof of this Theorem appeals to the celebrated Perron-Frobenius theorem whose basics we recount below [16, p. 536].

Theorem 5: Suppose a matrix $A \in \mathbb{R}^{n \times n}$ is nonnegative and irreducible. Then the largest eigenvalue of $A$ is simple, positive and has a corresponding eigenvector all whose elements are positive. If $A$ is reducible then the largest eigenvector corresponding to its largest eigenvalue can be chosen to be non-negative. 4.

We also need the following lemma to prove Theorem

Lemma 10: Suppose $A=A^{T} \neq B=B^{T}$ are nonnegative, irreducible, real matrices, and $B-A$ is a nonzero, non-negative matrix. Then: $\lambda_{\max }(A)<\lambda_{\max }(B)$.

Proof: Observe at least one element of $B-A$ is positive. From Theorem 5, $x \in \mathbb{R}^{n}$, the eigenvector corresponding to the largest eigenvalue of $A$ can be chosen to have all elements positive. Then the result follows from the fact that:

$$
\begin{aligned}
\lambda_{\max }(A) x^{T} x & =x^{T} A x \\
& =x^{T} B x-x^{T}(B-A) x \\
& <x^{T} B x \\
& \leq \lambda_{\max }(B) x^{T} x
\end{aligned}
$$

as $B-A$ is a non-zero, non-negative matrix.

Turning to the proof of Theorem 4 we note that the result follows directly from Lemma 10 and the fact $Q_{G^{\prime}}$ and $Q_{G}$ satisfy the requirements of $B$ and $A$, respectively.

\section{REFERENCES}

[1] P. Gupta and P. R. Kumar, "Critical power for asymptotic connectivity in wireless networks," in Stochastic Analysis, Control, Optimization and Applications. Boston, MA: Birkhauser, 1998, pp. 547-566.

[2] M. Haenggi, J. G. Andrews, F. Baccelli, O. Dousse, and M. Franceschetti, "Stochastic geometry and random graphs for the analysis and design of wireless networks," IEEE Journal on Selected Areas in Communications, vol. 27, no. 7, pp. 1029-1046, 2009.

[3] M. D. Penrose, Random Geometric Graphs, ser. Oxford Studies in Probability. Oxford University Press, USA, 2003.

[4] G. Mao and B. D. Anderson, "Connectivity of large scale networks: Emergence of unique unbounded component," submitted to IEEE Transactions on Mobile Computing, available at http://arxiv.org/abs/1103.1991. 2011.

[5] - "Connectivity of large scale networks: Distribution of isolated nodes," submitted to IEEE Transactions on Mobile Computing, available at http://arxiv.org/abs/1103.1994. 2011.

[6] R. Meester and R. Roy, Continuum Percolation, ser. Cambridge Tracts in Mathematics. Cambridge University Press, 1996.

[7] G. Mao and B. D. Anderson, "Towards a better understanding of large scale network models," accepted to appear in IEEE/ACM Transactions on Networking, 2011.

[8] L. Hogben, Handbook of Linear Algebra, ser. Discrete Mathematics and Its Applications. Chapman \& Hall/CRC, 2007.

[9] N. L. Biggs, Algebraic Graph Theory. Cambridge University Press, 1974.

[10] B. Mohar, "Laplace eigenvalues of graphs - a survey," Journal of Discrete Mathematics, vol. 109, pp. 171-183, 1992.

[11] C. Godsil and G. F. Royle, Algebraic Graph Theory. Springer, 2001.

[12] R. R. Brooks, B. Pillai, S. Racunas, and S. A. R. S. Rai, "Mobile network analysis using probabilistic connectivity matrices," IEEE Transactions on Systems, Man, and Cybernetics, Part C: Applications and Reviews, vol. 37, no. 4, pp. 694-702, 2007.

[13] G. Mao and B. D. Anderson, "Graph theoretic models and tools for the analysis of dynamic wireless multihop networks," in IEEE WCNC, 2009, pp. 1-6.

[14] J. v. d. Berg and H. Kesten, "Inequalities with applications to percolation and reliability," Journal of Applied Probabilities, vol. 22, pp. 556-569, 1985.

[15] C. Borgs, J. Chayes, and D. Randall, "The van den berg-kestenreimer inequality: A review," Tech. Rep. MSR-TR-98-42, 1998.

[16] P. Lancaster and M. Tismenetsky, The Theory of Matrices, ser. Computer Science and Applied Mathematics. Academic Press, 1985.

[17] L. Zadeh and C. A. . Desoer, Linear Systems Theory. McGraw Hill, New York, 1963.

[18] S. Dasgupta, C. Chockalingam, M. Fu, and B. Anderson, "Lyapunov functions for uncertain systems with applications to the stability of time varying systems," IEEE Transactions on Circuits and Systems-I, Fundamental Theory and Applications, vol. 41, no. 2, pp. 93-105, 1994. 